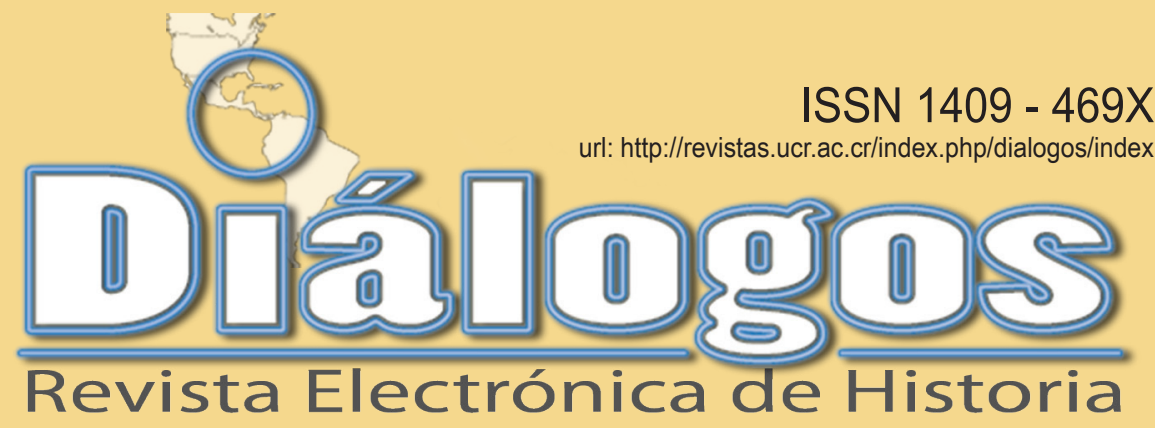

Escuela de Historia. Universidad de Costa Rica Volumen 15 Especial Región Occidente de Costa Rica - Octubre, 2014

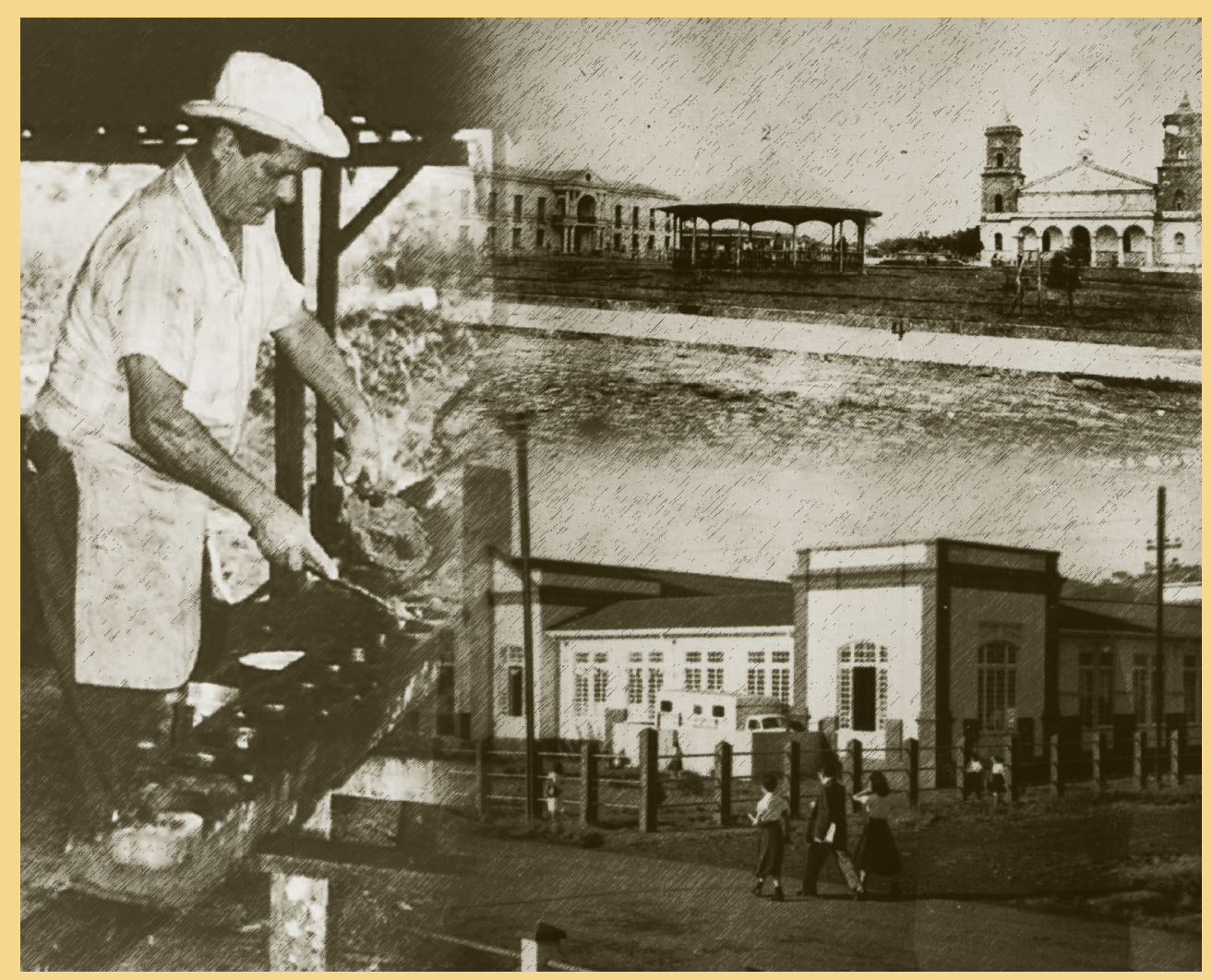

Director de la Revista: Dr. Juan José Marín Hernández juan.marinhernandez@ucr.ac.cr

Editor académico: Dr. David Díaz Arias - david.diaz@ucr.ac.cr

Editores invitados: M.Sc. William Solórzano Vargas - william.solorzano@ucr.ac.cr

M.Ed. Maynor Badilla Vargas - mainor.badilla@ucr.ac.cr

Editora técnica: M.Sc. Marcela Quirós G. - marcela.quiros@ucr.ac.cr 
Dr. Juan José Marín Hernández, Catedrático. Director del Centro de Investigaciones Históricas de América Central. Universidad de Costa Rica. Costa Rica. juan. marin@ucr.ac.cr

Dr. David Díaz Arias: Catedrático. Historia Política, Director del posgrado de Historia y Docente de la Escuela de Historia, Universidad de Costa Rica, Costa Rica.david.diaz@ucr.ac.cr

Dr. Ronny Viales Hurtado. Catedrático. Historia Económica y Social. Universidad de Costa Rica. Director de la Escuela de Historia. Costa Rica. ronny. viales@ucr.ac.cr

MSc. Francisco Enríquez. Historia Social. Universidad de Costa Rica. Costa Rica. francisco.enriquez@ucr. ac.cr

Dra. Ana María Botey. Historia de los movimientos sociales. Universidad de Costa Rica. Costa Rica. abotey@gmail.com

\section{Miembros del Consejo Asesor Internacional:}

Dr. José Cal Montoya. Universidad de San Carlos de Guatemala. Guatemala. jecalm@correo.url.edu.gt

Dr. Juan Manuel Palacio. Universidad Nacional de San Martín. Argentina.jpalacio@unsam.edu.ar

Dr. Eduardo Rey. Universidad de Santiago de Compostela. España. ereyt@usc.es

Dr. Heriberto Cairo Carou. Departamento de Ciencia Política y de la Administración III - Universidad Complutense de Madrid. España. hcairoca@cps.ucm.es

Dra. Rosa de la Fuente. Departamento de Ciencia Política y de la Administración III Universidad Complutense de Madrid. España. rdelafuente@cps. ucm.es

Dr. Javier Franzé. Departamento de Ciencia Política y de la Administración III Universidad Complutense de Madrid. España. javier.franze@cps.ucm.es

Dr. Jaime Preciado Coronado Departamento de Estudios Ibéricos y Latinoamericanos. Universidad de Guadalajara. México.japreco@hotmail.com

Dr. Gerónimo de Sierra. Vicerrector de la Universidade Federal da Integração Latino-Americana (UNILA) y Departamento de Sociología, Facultad de Ciencias

Sociales de la Universidad de la República. Uruguay. geronimo@fcs.edu.uy

Dr. Antonio Palazuelos. Departamento de Ciencia Política y de la Administración III - Universidad Complutense de Madrid. España. palazuelosa@cps. ucm.es

Dr. Werner Mackenbach. Universidad Potsdam. Alemania.werner.mackenbach@uni-potsdam.de

Dr. Guillermo Castro. Ciudad del Saber Panamá. Panamá.gcastro@cdspanama.org

Dra. Natalia Milanesio. University of Houston. Estados Unidos.nmilane2@Central.UH.EDU

Dr. Ricardo González Leandri. Consejo Superior de Investigaciones Científicas - España. España. rgleandri@gmail.com

Dra. Mayra Espina. Centro de Estudios Psicológicos y Sociológicos, La Habana. Cuba.mjdcips@ceniai.inf.cu

Dra. Montserrat Llonch. Departamento de Economía e Historia Económica Universidad Autónoma de Barcelona. España. montserrat.llonch@uab.es

Dra. Estela Grassi. Universidad de Buenos Aires. Argentina. estelagrassi@gmail.com

Dra. Yolanda Blasco. Universidad de Barcelona. España. yolandablasco@ub.edu

Dr. Alfredo Falero. Departamento de Sociología. Universidad de la República. Uruguay. alfredof@adinet. com.uy

Portada:

Fotografía: ( Collage "Campesinos en la producción de dulce en un trapiche de Rincón de Mora, Ran Ramón, 1987" ; "Panorámica del Antiguo Palacio Municipal, Antigua Iglesia, Parque y Kiosco de San Ramón, hacia 1920; y Escuela Jorge Washinton, San Ramón hacia 1955). Estas fotografías pertenecen a la Colección Fotográfica del Museo Regional de San Ramón- UCR. Montaje fotográfico: Lic. Juan Gabriel Madrigal Cubero ).

\section{Equipo Técnico Editorial:}

Diagramación y

Edición técnica:

M.Sc. Marcela Quirós Garita. marcela.quiros@ucr.ac.cr

Soporte técnico: Kevin Trejos Vargas

Revisión filológica: Baruc Chavarría Castro 
"Diálogos Revista Electrónica de Historia" se publica desde octubre de 1999.

\section{Diálogos está en los siguientes repositorios:}

Dialnet

http://dialnet.unirioja.es/servlet/

revista?tipo_busqueda=CODIGO\&clave_revista $=3325$

\section{Latindex}

http://www.latindex.unam.mx/larga.php?opcion=1\&folio=12995;

\section{UCRindex}

http://www.revistas.ucr.ac.cr

\section{Scielo}

http://www.scielo.cll

\section{eRevistas}

http://www.erevistas.csic.es/

\section{REDALYC}

http://redalyc.uaemex.mx/src/inicio/FrmBusRevs2.jsp?iEdoRev=2\&cvepai=11;

\section{LANIC}

http://lanic.utexas.edu/la/ca/cr/indexesp.html;

Repositorio de Revistas Universidad de Costa Rica

http://www.latindex.ucr.ac.cr/

Directorio y recolector de recursos digitales del

Ministerio de Cultura de España

http://roai.mcu.es/es/inicio/inicio.cmo

DOAJ Directory of open access \& Hybrid journals

http://www.doaj.org/doaj?func=byTitle\&hybrid=1\&query=D

Biblioteca de Georgetown

http://library.georgetown.edu/newjour/d/msg02735.htm

Asociación para el Fomento de los Estudios Históricos en Centroamérica

http://afehc.apinc.org/index.php?action=fi_aff\&id=1774

Universidad de Saskatchewan, Canadá

https://library.usask.ca/ejournals/view/1000000000397982

Monografias

http://www.monografias.com/Links/Historia/more12.shtml

\section{Hispanianova}

http://hispanianova.rediris.es/general/enlaces/hn0708.htm

Universidad del Norte, Colombia

http://www.uninorte.edu.co/publicaciones/memorias/enlaces.htm

Universidad Autónoma de Barcelona

http://seneca.uab.es/historia/hn0708.htm

Repositorio Invenia - Gestión del Conocimiento http://www.invenia.es/oai:dialnet.unirioja.es:ART0000086144

\section{Enlace Académico}

http://www.enlaceacademico.org/biblioteca/

revistas-en-formato-digital-centroamerica/

\section{Electronic Resources}

http://sunzi1.lib.hku.hk/ER/detail/hkul/3987318

Revistas académicas en texto completo http://web.prw.net/ vtorres/

Diálogos se anuncia en las siguientes instituciones y sitios académicos:

Maestroteca

http://www.maestroteca.com/detail/553/dialogos-revista-electronica-de-historia.html

Biblioteca de Georgetown

http://library.georgetown.edu/newjour/d/msg02735.htm

Asociación para el Fomento de los Estudios Históricos en Centroamérica

http://afehc.apinc.org/index.php?action=fi_aff\&id=1774

Universidad de Saskatchewan, Canadá

https://library.usask.ca/ejournals/view/1000000000397982

Monografias

http://www.monografias.com/Links/Historia/more12.shtm

Hispanianova

http://hispanianova.rediris.es/general/enlaces/hn0708.htm

Universidad del Norte, Colombia

http://www.uninorte.edu.co/publicaciones/memorias/enlaces.html

Universidad Autónoma de Barcelona

http://seneca.uab.es/historia/hn0708.htm

Repositorio Invenia - Gestión del Conocimiento

http://www.invenia.es/oai:dialnet.unirioja.es:ART0000086144

Enlace Académico

http://www.enlaceacademico.org/biblioteca/

revistas-en-formato-digital-centroamerical

\section{Electronic Resources}

http://sunzi1.lib.hku.hk/ER/detail/hkul/3987318

Revistas académicas en texto completo http://web.prw.net/ vtorres/

Diálogos Revista de Historia está catalogada por Sherpa Romeo como una revista verde.

La revista electrónica Diálogos es financiada por Vicerrectoría de Investigación de la Universidad de Costa Rica

Citado en: Scielo Dialnet - eRevistas - UCRindex Latindex - REDALYC - DOAJDirectorio y recolector de recursos digitales del Ministerio de Cultura de España

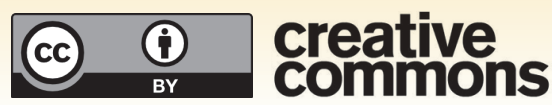


Segunda parte

Socioeconomía 



\title{
APROXIMACIONES AL ESTUDIO DE LA POBREZA EN LA REGIÓN DE OCCIDENTE: LOS CANTONES DE NARANJO, PALMARES Y SAN RAMÓN
}

\section{APPROACHES TO THE STUDY OF POVERTY IN THE WESTERN REGION: THE CANTONS OF NARANJO, PALMARES AND SAN RAMÓN}

\author{
Illiana Arroyo Navarro \\ Melba Rodríguez Rodríguez
}

\begin{abstract}
Palabras claves
Pobreza, estrategias de sobrevivencia, familia.
\end{abstract}

Keywords

Poverty, survival strategies, family.

Fecha de recepción: 23 de mayo, 2014 - Fecha de aceptación: 28 de julio, 2014

\section{Resumen}

El objeto de investigación propuesto en este artículo está dirigido a identificar las modificaciones que se han generado en las estructuras familiares a partir de la puesta en marcha de estrategias ante la crisis actual; específicamente, en los cantones de Naranjo, Palmares y San Ramón. El impacto del estudio se orienta a aportar al conocimiento de la situación familiar de la Región de Occidente de Costa Rica en el contexto de la crisis del capital.

\begin{abstract}
The object of the investigation proposed in this paper is aimed at identifying the changes that have resulted in family structures from the implementation of strategies, the current crisis, specifically in the cantons of Naranjo, Palmares y San Ramon. The impact of the study is designed to contribute to the knowledge of the family situation of the Western Region of Costa Rica in the context of the crisis of capital.
\end{abstract}




\section{APROXIMACIONES AL ESTUDIO DE LA POBREZA EN LA REGIÓN DE OCCIDENTE: LOS CANTONES DE NARANJO, PALMARES Y SAN RAMÓN}

\section{INTRODUCCIÓN}

La Región de Occidente se ha visto inmersa en una serie de cambios sociales, económicos, políticos y culturales, que se expresan de diversas formas y configuran un contexto cada vez más importante para investigar.

Este artículo se inscribe dentro del contexto de una investigación que se está desarrollando en la Sede de Occidente específicamente desde la carrera de Trabajo Social de la Universidad de Costa Rica en el Programa de Desarrollo Regional y que lleva como título: "Estrategias de Sobrevivencia y Reconfiguraciones Familiares en la Región de Occidente".

Con la finalidad de aportar a la discusión sobre el tema de la pobreza, se parte de algunas premisas que pretenden contextualizar la pertinencia del abordaje de dicha cuestión en la Región de Occidente.

Las transformaciones sociales, culturales y económicas a partir del nuevo orden mundial han generado en Costa Rica un proceso de agudización de la pobreza. Se ha encontrado que cada vez más sectores de la población han pasado de una pobreza general - es decir, lo que el Programa de Naciones Unidas para el Desarrollo (PNUD) denomina el nivel menos grave de pobreza, que es entendido normalmente como incapacidad de atender las necesidades alimentarias y no alimentarias esenciales - , a niveles de pobreza absoluta: pobreza entendida de acuerdo con un criterio fijo. Un ejemplo sería el umbral internacional de un dólar diario, cuyo objetivo es comparar el alcance de la pobreza en distintos países. En muchos casos, este término se utiliza también en sentido menos riguroso para referirse a la pobreza extrema (Programa de Naciones Unidas para el Desarrollo, 1998, p. 16).

Según el Instituto Nacional de Estadística y Censos (2010), para el año 2008 los hogares en condición de pobreza constituían el 17,7\% y para 2009 fue del 18,5\%. Así también la pobreza extrema o absoluta pasó del 3,5\% en 2008 al 4,2\% en 2009.

Es importante señalar que los indicadores estadísticos expresan claramente esa brecha social, como son los referidos a los índices de ingresos que tienden a un estancamiento. Tal es el caso del coeficiente de Gini ${ }^{1}$ que pasa de 0,426 en 2007 a 0,421 en 2008, cifra igual al promedio del periodo 2000-2007; es decir, refleja la desigualdad en la distribución de los ingresos que ha prevalecido en la presente década (Programa Estado de la Nación en Desarrollo Humano Sostenible, 2009, p. 26). Por su parte, según datos de del Ministerio de Planificación Nacional y 
Política Económica (Mideplan), para 2009 el coeficiente de Gini alcanzó el rango más elevado en la historia de Costa Rica llegando a 0,439. Esta inequidad en la distribución de los ingresos demuestra claramente el contexto de pobreza en que se están moviendo las familias.

La pobreza se ha estudiado y medido desde diferentes enfoques, siempre íntimamente vinculada a la satisfacción de las necesidades básicas familiares. Son varias las concepciones que se han construido en torno a esta categoría teórica; por ejemplo, la planteada por Menjívar en 1990 permite un mejor acercamiento, permitiendo comprenderla dentro de su complejidad. Menjívar define pobreza como: la presencia de niveles de vida o bienestar socialmente inaceptables. Esta inaceptabilidad puede aludir a una dimensión absoluta del fenómeno; es decir, corresponde a situaciones en donde está en juego la propia existencia del individuo o puede tener un carácter más relativo, esto es, referirse a condiciones de marginación con relación a los niveles medios de vida que ostenta la sociedad específica en un tiempo dado (Menjívar y Trejos, 1990, p. 15).

Para acercarse al tema se presenta una sistematización de las definiciones de pobreza:

- Pobreza humana: la que se define como carencia de las capacidades humanas esenciales, como la alfabetización y una nutrición suficiente.

- Pobreza extrema: indigencia o miseria, normalmente entendida como incapacidad de atender ni siquiera las necesidades alimentarias mínimas.

- Pobreza general: se concibe y aplica para referirse al nivel menos grave de pobreza, entendido normalmente como incapacidad de atender las necesidades alimentarias y no alimentarias esenciales, cabe aclarar que la definición de necesidades no alimentarias esenciales puede variar significativamente de unos países a otros.

- Pobreza relativa: esta definición es entendida de acuerdo con criterios que pueden cambiar entre distintos países y a lo largo del tiempo. Un ejemplo sería el umbral de la pobreza establecido en la mitad del ingreso medio per cápita, lo que significa que el umbral puede subir a medida que suban también los ingresos. En muchos casos, este término se utiliza en sentido menos riguroso como sinónimo de pobreza general.

- Pobreza absoluta: esta categoría parte de entender la pobreza de acuerdo con un criterio fijo. Un ejemplo sería el umbral internacional de un dólar diario, cuyo objetivo es comparar el alcance de la pobreza en distintos países. Otro ejemplo es un umbral de pobreza cuyo valor real se mantenga sin cambios a lo largo del tiempo con el fin de determinar la evolución de la pobreza en un determinado país. En muchos casos, este término se utiliza también en 
sentido menos riguroso para referirse a la pobreza extrema (Programa de Naciones Unidas para el Desarrollo, 1998, p. 16).

Dentro de esta exposición también se considera importante lo que se ha denominado los nuevos pobres: teóricamente los nuevos pobres son sectores de población que recientemente han caído por debajo de la línea de pobreza y que han debido ejecutar acciones de emergencia para satisfacer sus necesidades. Otros grupos de la clase media han debido también desarrollar estrategias de sobrevivencia nuevas a partir del deterioro de sus ingresos, pero en tanto permanecen por encima de la línea de pobreza no son considerados pobres aunque se encuentran en proceso de "empobrecimiento" (Sojo, 1997, p. 15).

Tradicionalmente la pobreza se ha medido mediante parámetros lo que ha llevado a reducirla a indicadores estadísticos como instrumentos para medirla. Dentro de las mediciones de pobreza se exponen dos métodos utilizados frecuentemente:

Método de ingreso: la primera aproximación consiste en poner la atención en los insumos que requieren las familias para satisfacer sus necesidades y en particular en el ingreso como insumo clave. Este método indirecto también conocido como método del ingreso o de la línea de pobreza, consiste en principio en los siguientes pasos:

- Definición de una canasta normativa de satisfactores esenciales para cada hogar.

- Cálculo del costo de esa canasta básica que se constituye en la línea de pobreza.

- Comparación de la línea de pobreza con el ingreso del hogar.

- Clasificación de todos los hogares y sus miembros, cuyo ingreso es menor que la línea de pobreza como pobres.

El método de necesidades básicas insatisfechas: la segunda aproximación consiste en los resultados finales del proceso de satisfacción de las necesidades de las familias, esto es, en el consumo efectivo de un conjunto de bienes y servicios considerados como básicos. Este método directo, también denominado método de necesidades básicas insatisfechas consiste, en principio, en los siguientes pasos:

- Identificación de las necesidades que se consideran como básicas;

- Selección de las variables e indicadores que, para cada necesidad, expresan el grado de su satisfacción;

- Definición de un nivel mínimo para cada indicador, debajo del cual se considera que el hogar no satisface la necesidad en cuestión, y clasificación de todos los hogares, y sus miembros, con una o más necesidades insatisfechas como pobres (Menjívar y Trejos, 1990, pp. 22-24). 
Estos métodos permiten un acercamiento al fenómeno de la pobreza que, si bien de carácter cuantitativo, admite dimensionar y orientar política. También los organismos internacionales han desarrollado procedimientos metodológicos para medir la pobreza, retomando lo que plantea Carlos Sojo (1997, p. 14):

- Modelo de la Comisión Económica para América Latina (CEPAL): basado en una línea de pobreza definida por el costo de una canasta alimentaria conformada a partir de la necesidad teórica de consumo calórico de una unidad consumidora.

- Modelo del Banco Interamericano de Desarrollo (BID): que agrega una medición de necesidades alimentarias ponderadas por edad y sexo, e incluye consumo no alimentario para la fijación de la línea de pobreza.

- $\quad$ Necesidades Básicas Insatisfechas (NBI): este no establece línea de ingreso pues determina el umbral de la pobreza a partir del consumo efectivo de ciertos servicios básicos como vivienda, salud y educación.

\section{ALGUNOS ÍNDICES SOCIODEMOGRÁFICOS PRESENTES EN LOS CANTONES DE SAN RAMÓN, PALMARES Y NARANJO.}

Los índices demográficos o estadísticos constituyen datos que permiten conocer un fenómeno y sirven como punto de partida para estudiar comparativamente problemáticas particulares. Entre los índices que se han empleado para el estudio de la pobreza en cantones se retoman los expuestos por la Escuela de Estadística y el Observatorio del Desarrollo, los que se exponen a continuación:

El Índice de Desarrollo Humano Cantonal (IDH) se compone de: Índice de Esperanza de Vida (IEV), Índice de Conocimiento (IC) e Índice de Bienestar Material (IBM), sus valores se encuentran entre 0 y 1 , cuanto más cercano a 1 sea el valor entonces se tiene un mejor IDH. Se compararon datos para el periodo 1999 a 2006.

El otro Índice consultado fue el de Pobreza Humana (IPH) se compone de: los indicadores Probabilidad al nacer de no sobrevivir a los 60 años, Porcentaje de adultos que cuentan con nivel educativo menor de tercer grado, Porcentaje de personas pobres y Tasa de desempleo a largo plazo. Sus valores se encuentran entre 0 y 100 la información a la que se tuvo acceso fue para los años de 1999 a 2006. Con los datos analizados se tiene que tanto San Ramón como Palmares muestran una tendencia a la disminución (IPH), mientras que en el caso de Naranjo se muestra un aumento de dicho índice (ver Tablas 1, 2 y 3). 


\section{Tabla 1}

INDICADORES DE POBREZA DEL CANTÓN DE SAN RAMÓN SEGÚN

TENDENCIA HISTÓRICA 1999-2008

\begin{tabular}{lcc}
\hline INDICADORES & 1999 & 2008 \\
\hline Índice de Bienestar Material & 0,40 & 0,46 (dato a 2006) \\
Índice de Pobreza Humana & 11,07 & 10,96 (dato a 2006) \\
\hline
\end{tabular}

Fuente: Elaboración propia con base en www.odd.ucr.ac.cr/

\section{Tabla 2}

INDICADORES DE POBREZA DEL CANTÓN DE NARANJO SEGÚN TENDENCIA HISTÓRICA 1999-2008

\begin{tabular}{lcc}
\hline INDICADORES & 1999 & 2008 \\
\hline Índice de Bienestar Material & 0,42 & 0,46 (dato a 2006) \\
Índice de Pobreza Humana & 10,54 & 10,93 (dato a 2006) \\
\hline
\end{tabular}

Fuente: Elaboración propia con base en www.odd.ucr.ac.cr/

Tabla 3

INDICADORES DE POBREZA DEL CANTÓN DE PALMARES SEGÚN

TENDENCIA HISTÓRICA 1999-2008

\begin{tabular}{lcc}
\hline INDICADORES & 1999 & 2008 \\
\hline Índice de Bienestar Material & 0,50 & 0,50 (dato a 2006) \\
Índice de Pobreza Humana & 11,50 & 10,47 (dato a 2006) \\
\hline
\end{tabular}

Fuente: Elaboración propia con base en www.odd.ucr.ac.cr/ 


\section{LA DISTRIBUCION Y USO DEL SUELO UNA FORMA PARA EXPLICAR LOS CAMBIOS DE LOS CANTONES ESTUDIADOS.}

La Región de Occidente, en la que se ubican los cantones de San Ramón, Palmares y Naranjo, es una zona de transición, cuya actividad económica históricamente ha estado vinculada a la agricultura de café, tabaco, hortalizas y a la ganadería de leche principalmente. Sin embargo, la crisis económica y los procesos de urbanización cada vez más acelerados han dado lugar a cambios en las actividades productivas de la región.

Así se tiene que una de las modificaciones más importantes en cuanto a la estructura productiva y que permite medir la transacción es el uso del suelo. Por lo que se indagó sobre los permisos otorgados para la construcción de áreas destinadas al comercio, la industria y residencial en los cantones estudiados.

En el caso del cantón de San Ramón, los permisos otorgados para la construcción de áreas consignadas al comercio pasaron de $4.552 \mathrm{~m}^{2}$ en 2001 a $23.427 \mathrm{~m}^{2}$ en 2007. También se presenta un leve aumento en los espacios destinados a servicios, en contraposición a los propuestos para industria y actividades agropecuarias que presentan una disminución importante sobre todo en los suelos agrícolas. Se destaca el aumento de las superficies destinadas a viviendas, que en un periodo de 7 años triplicó los metros cuadrados (ver Tabla 4).

Dentro de esta misma línea de análisis se tiene que el cantón de Palmares ha experimentado cambios importantes en el uso y distribución del suelo. De acuerdo a los datos consultados en cuanto a metros cuadrados de construcción por permiso se destacan, en primer lugar, un sostenido aumento de las superficies destinadas a vivienda, a partir de la tendencia urbanizante que ha enfrentado La Región de Occidente, específicamente los cantones de San Ramón, Palmares y Naranjo, es una zona de transición, cuya actividad económica históricamente ha estado vinculada a la agricultura de café, tabaco, hortalizas y a la ganadería de leche principalmente. Estas actividades históricamente han caracterizado a la zona; sin embargo, la crisis económica y los procesos de urbanización cada vez más acelerados han dado lugar a cambios en las actividades productivas de la región.

La región ha ido presentando modificaciones importantes en cuanto a la estructura productiva. Una forma de acercarse a este cambio tiene que ver con la forma en la distribución y uso del suelo, dándose modificaciones significativas en los sitios destinados a diferentes actividades.

En el caso del cantón de Naranjo presenta características similares a los cantones antes descritos; sin embargo, se destaca una tendencia a mantener lo agropecuario como actividad productiva principal, pasando en 2001 de 356 a 2.986 en 
2007. No obstante, muestra en común el incremento importante en las áreas para vivienda (ver Tabla 6).

Estos cambios en la distribución y utilización del suelo han ido modificando las actividades productivas en los cantones, reconfigurando una nueva estructura social, y demográfica y por ende económica en donde se dan nuevas articulaciones familiares y expresiones de deterioro en las condiciones de vida de estas.

\section{Tabla 4}

INDICADORES DE DISTRIBUCIÓN DEL SUELO EN RELACIÓN CON LA ACTIVIDAD PRODUCTIVA DEL CANTÓN DE SAN RAMÓN

\begin{tabular}{ccc}
\hline $\begin{array}{c}\text { ÁREAS EN METROS CUADRADOS } \\
\text { DE CONSTRUCCIÓN POR PERMISO }\end{array}$ & 2001 & 2007 \\
\hline Comercio & 4.552 & 23.426 \\
Servicios & 390 & 436 \\
Vivienda & 26168 & 96097 \\
Industria & 654 & 351 \\
& & 13.111 \\
\hline
\end{tabular}

Fuente: Elaboración propia con base en www.odd.ucr.ac.cr/

\section{Tabla 5}

INDICADORES DE DISTRIBUCIÓN DEL SUELO EN RELACIÓN CON

LA ACTIVIDAD PRODUCTIVA DEL CANTÓN DE PALMARES.

\begin{tabular}{ccc}
\hline $\begin{array}{c}\text { ÁREAS EN METROS CUADRADOS } \\
\text { DE CONSTRUCCIÓN POR PERMISO }\end{array}$ & 2001 & 2007 \\
\hline Comercio & 3.845 & 5.706 \\
Servicios & 180 & 175 (dato a 2005) \\
Vivienda & 19.604 & 21.824 \\
Industria & 1.974 & 246 \\
Agropecuario & 166 & 161 \\
\hline
\end{tabular}

Fuente: Elaboración propia con base en www.odd.ucr.ac.cr/ 


\section{Tabla 6}

INDICADORES DE DISTRIBUCIÓN DEL SUELO EN RELACIÓN CON

LA ACTIVIDAD PRODUCTIVA DEL CANTÓN DE PALMARES.

\begin{tabular}{ccc}
\hline $\begin{array}{c}\text { ÁREAS EN METROS CUADRADOS } \\
\text { DE CONSTRUCCIÓN POR PERMISO }\end{array}$ & 2001 & 2007 \\
\hline Comercio & 1684 & 3215 \\
Servicios & 154 & 97 \\
Vivienda & 14973 & 20800 \\
Industria & 63 & 803 \\
Agropecuario & 356 & 2986 \\
\hline
\end{tabular}

Fuente: Elaboración propia con base en www.odd.ucr.ac.cr/

\section{CONCLUSIONES}

La globalización trajo consigo un deterioro en las condiciones de vida de la población costarricense, ya debilitada por las políticas de ajuste de la década de los ochenta, rompiendo con los esquemas de la pequeña producción agrícola y artesanal propia de la Región de Occidente para dar lugar a un proceso de exclusión social y pobreza.

Los cantones de San Ramón, Palmares y Naranjo de la provincia de Alajuela se han caracterizado por economías históricamente vinculadas al sector agrícola; no obstante, en las últimas décadas ha ido incursionando cada vez más en actividades relacionadas con los servicios y el comercio. Estas transformaciones en la estructura productiva han dado lugar a un proceso de empobrecimiento paulatino. Dentro del contexto de incertidumbre, las familias de la región siguen cumpliendo sus funciones en escenarios cada vez más empobrecidos y desempeñando los roles establecidos socialmente dentro de condiciones más adversas. Los ajustes o arreglos familiares constituyen expresiones de los cambios socio-demográficos, culturales y económicos, lo que puede suponer nuevas configuraciones familiares que respondan a esas transformaciones.

Los cambios a nivel estructural nacional y específicamente en la región han dado lugar a que las familias de los cantones de San Ramón, Naranjo y Palmares, enfrenten modificaciones sociodemográficas, económicas y culturales. Estos cambios 
pueden ser identificados desde diferentes fuentes de información e indicadores. En ese sentido, al referirse al tema Ariza y Oliveira señalan que:

\footnotetext{
"Se trata de transformaciones de largo alcance que rebasan, sin duda, al mundo familiar, pero que constituyen el preámbulo necesario para comprender el cuestionamiento actual de algunos de los presupuestos teóricos que guían su estudio. Tales cambios engloban tanto la dinámica socioeconómica, como la demográfica y la sociocultural, con consecuencias disímiles para las familias y quienes las integran.” (Ariza y Oliveira, 2001, p. 11)
}

Es decir, no solamente se trata de analizar los datos, sino de interpretarlos en la complejidad de las transformaciones socioeconómicas y demográficas.

Mediante los permisos de construcción otorgados se logra visualizar la forma como se han ido redistribuyendo las áreas dedicadas a diferentes actividades en los cantones estudiados, encontrando un aumento significativo en los terrenos destinados a vivienda; por ejemplo, el número manifiesto de proyectos habitacionales que han proliferado en San Ramón y Palmares principalmente. Por otro lado, el incremento de los permisos orientados al comercio y los servicios que explican las modificaciones en la estructura sociodemográfica y productiva de los cantones.

Hablar de pobreza desde una visión cuantitativa, si bien no logra la totalidad conceptual que requiere esta categoría teórica, permite un acercamiento a la temática desde los índices e indicadores, en tanto señalan las tendencias y proyecciones para orientar las políticas y acciones para el tratamiento de la pobreza.

\section{CITAS Y NOTAS}

1. Coeficiente de Gini: es una medida de desigualdad en la distribución del ingreso, su valor oscila entre 0 y 1 , a medida que el índice se acerca a la unidad, la concentración se hace cada vez mayor.

\section{REFERENCIAS}

Ariza, M. y Oliveira, O. (2001). Familias en transición y marcos conceptuales en redefinición. Papeles de Población, 7(28), 9-39. Recuperado de http://www.redalyc.org/articulo. oa? id $=11202802$ 
Instituto Nacional de Estadística y Censos. (2000) IV Censo 2000. Costa Rica: Instituto Nacional de Estadística y Censos.

Menjívar, R y Trejos, J. (1990). La pobreza en América Central. Costa Rica: FLACSO.

Programa Estado de la Nación. (2009). Decimoquinto Informe Estado de la Nación en Desarrollo Humano Sostenible. Costa Rica: Programa Estado de la Nación.

Programa de las Naciones Unidas para el Desarrollo (1998). Superar la pobreza humana. Nueva York: Programa de las Naciones Unidas para el Desarrollo.

Sojo, C. (1997). Los de en medio: la nueva pobreza en Costa Rica. Costa Rica: FLACSO.

Universidad de Costa Rica. (2010). Observatorio del Desarrollo. Recuperado de www.odd.ucr.ac.cr/

\section{ACERCA DE LAS AUTORAS}

Illiana Arroyo Navarro. Docente e investigadora de la carrera de Trabajo Social de la Universidad de Costa Rica, Sede de Occidente. Correo electrónico: illianaarroyo0@gmail.com

Melba Rodríguez Rodríguez. Docente e investigadora de la carrera de Trabajo Social de la Universidad de Costa Rica, Sede de Occidente. Correo electrónico: melba.rodriguezrodriguez1@ gmail.com 
